\title{
ANCIENT STONE TIDAL WEIRS IN PENGHU ARCHIPELAGO: DISTRIBUTION, CATEGORY, STRUCTURE AND FUNCTION, A GOOGLEEARTH AND GIS APPROACH
}

\author{
L. Luo ${ }^{\text {a, b* }}$, X. Y. Wang ${ }^{\text {a, b }}$, J. Liu ${ }^{\text {a, b }}$, H. D. Guo ${ }^{\text {a, b }}$ \\ ${ }^{a}$ International Centre on Space Technologies for Natural and Cultural Heritage under the Auspices of UNESCO, 100094 Beijing, \\ China - (luolei, wangxy, liujie, hdguo)@ radi.ac.cn \\ ${ }^{\mathrm{b}}$ Institute of Remote Sensing and Digital Earth, Chinese Academy of Sciences, 100094 Beijing, China
}

Commission VI, WG VI/4

KEY WORDS: Stone tidal weirs, Google Earth, GIS, Penghu, Archaeological, Heritage

\begin{abstract}
:
The aim of this study was to give a comprehensive archaeological investigation for Penghu's stone tidal weirs (STWs) based on both Google Earth and GIS. Firstly, this study uses GoogleEarth Pro tools to clip a GeoEye-1 image (acquisition date: 22/07/2013) and a WorldView-2 image (acquisition date: 25/01/2014) for Chipei Island and Husi Island, respectively, and save them at a "premium resolution" of $4800 \mathrm{dpi}$. More, using $15 \mathrm{~m}$ panchromatic orthorectified Landsat images as a base, two clips were geo-referenced in ENVI 5.1 with minimal root mean square error. Furthermore, the STWs were manual extracted from the two GoogleEarth images in ArcGIS 10.1. Category and size statistics are presented; construction structure and weir function are discussed. Lastly, by using GIS analyses, STWs characteristics of intertidal flats across Penghu archipelago have been mapped and related to key geographical environmental variables. From spring to summer of 2015 our research team conducted investigations into Penghu's STWs based on different seasons and time periods of GoogleEarth historic images. Our results showed that, distributed amongst Penghu's coastline, there are 503 STWs. Compared with the official survey results (around 592 STWs), the counts are similar but the GoogleEarth-based method is more time-saving and efficient.
\end{abstract}

\section{INTRODUCTION}

Stone weirs have been effective for hundreds of years not only in Taiwan (both found in Taiwan Island and Penghu archipelago) but also placed on other pacific islands such as Japan, Philippine, Korea and Australia. The Penghu's STWs, the most densely populated weir area in the world, are listed as one of the most important cultural heritages in Taiwan. The history of STWs in Penghu can be traced back at least to the 18th century during Qing dynasty.

Several precise surveys have been conducted for Penghu's STWs during the past two decades, and two most valuable investigations were produced: the former is purely field surveys from 1996 to 1999 by Hong (1999), and the later is the Digital Archives Program (http://www.daweir.npu.edu.tw/) which integrated field works with aerial remote sensing and GIS (Li and Zhan, 2006) in the beginning of 21 st century. These two works found out the real situation of STWs in the entire Penghu archipelago, but are human, time, material and financial resources consuming and the Digital Archives Program strongly depend on the digitalization experience of investigators.

Over the last decade, GoogleEarth has provided an unprecedented variety of remotely sensed very high resolution imagery with a spatial resolution of $1 \mathrm{~m}$ or finer. Although it does not provide multispectral information, it allows for regional investigations (Sadr and Rodier, 2012; Kempe and AlMalabeh, 2013) even small archaeological sites and features to be detected (Luo et al., 2014), which is a huge step forward for archaeological purpose. GoogleEarth Pro is a business-oriented upgrade to GoogleEarth. It is the most feature-rich version of
GoogleEarth available to the public, with various additional features such as a movie maker and data importer. Google decided to make Google Earth Pro free to the public in January of 2015 .

GIS has over the last 10 years become an important tool in archaeology. The applications of GIS in archaeology are visualization, spatial analysis and archaeological predictive modeling (Luo et al., 2014). GIS-based archaeology is now paid more and more attention all over the world and there have been many successful cases which are mostly engaged in archaeological mapping, prospection and relic management (Espa et al., 2006; Evans et al., 2007; Alexakis et al., 2011; Sadr and Rodier, 2012).

The aim of this study is to present a case study to demonstrate how GoogleEarth and GIS have been able to contribute to the investigation, interpretation and documentation of STWs on the flats in Penghu archipelago. It is a time and money saving method for large scale geoarchaeological study.

\section{MATEIRAL AND METHODOLOGY}

\subsection{GoogleEarth images}

For the Penghu archipelago, GoogleEarth coverage includes 05meter color GeoEye-2 images from 2013 and WorldView images from 2014. The program has tools to clip fourteen GeoEye images (acquisition date: 22/07/2013) and three WorldView images (acquisition date: 25/01/2014), and save them at a "premium resolution" of 4800 dpi by using the GoogleEarth Pro.

\footnotetext{
* Corresponding author: luolei@radi.ac.cn.
} 
GoogleEarth images are only RGB renderings, so we are not looking for true DNs of the original images. In brief, they are not usable for quantitative remote sensing applications, but are still interesting for archaeological object recognition and visual applications (Luo et al., 2014). All image slices were clipped with each image covering a ground area of $900 \mathrm{~m} \times 600 \mathrm{~m}$. Using $15 \mathrm{~m}$ panchromatic orthorectified Landsat-8 OLI image as a base, all clips were geo-referenced in ArcGIS10.1 with minimal root mean square error. Upper image in Figure 1 shows the example of STWs visible on GoogleEarth VHR images with a resolution of $4800 \times 3277$ pixels.

\subsection{Manual extraction of STWs}

The primary objective was to create a map to understand the distribution, scale and structure of the STWs. Because GoogleEarth imagery has a high resolution, it is an ideal source for mapping this piscatorial landscape. Due to lack of a distinctive spectral signature for the linear archaeological trace of STWs in the imagery, (semi-) automatic feature extraction methods, combined image segmentation with edge detection, were not very successful except at extremely restricted spatial scales (Morehart, 2014).

In order to map the STWs, ArcGIS10.1 mapping tools were used to draw them from the clips. Lower image in Figure 1 shows the manual extraction results for the upper image with red polylines. The further analysis of STWs (statistic analysis, cluster analysis, classification and geometry calculation) will be operated after manual extraction by using spatial analysis tools under the ArcGIS10.1.
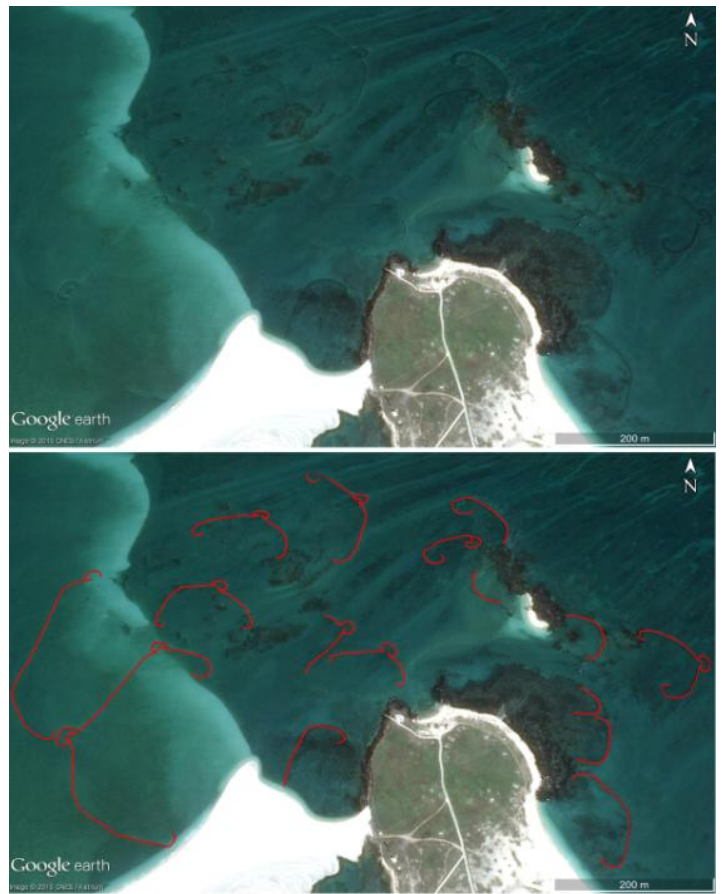

Figure 1. GeoEye-2 image (ㄷ GeoEye Image Copyright 2015, DigitalGlobe) from GoogleEarth Pro (upper) and red polylines indicate manual extractions of STWs (lower)

\subsection{Ground-truthing reference}

In our study, the Digital Archives Program (2006) (592 STWs together) was used as the reference catalog. By drawing a comparison to this ground-truthing database, the performance of our GoogleEarth-based method was evaluated.

\section{RESULTS}

\subsection{Categories and structure}

The STWs in Penghu archipelago can be divided into three main categories: Arched STWs (Figure 2 a), Single-room STWs (Figure $2 \mathrm{~b}$ ), and Double-room STWs (Figure $2 \mathrm{c}$ ). Generally, the Arched STWs, dustpan-shaped weirs, sit close to the shoreline, were built in shallower waters, and are thus often called "shallow-plain weirs", with "plain" indicating their having been smoothed into an abrasion platform by the waves of the intertidal zone $(\mathrm{Li}, 2009)$. The water level in Arched STWs was low or dry when tide falls. Only deeper places have water where fishermen can catch fish.

The Single-room and Double-room STWs, necklace-shaped weirs with one or two heart-like weir room, are often constructed in intertidal-flats that are quite far from the shoreline; owing to their deep water and remote locations, they are also known as "deep weirs" or "outer weirs". The primary function of the heart-like weir rooms is to collect and catch fish, and they often hold the deeper water than any other parts of the weir. Hence, as the tide falls, the fish have no choice but to swim into the weir rooms. In order to stop the fish from swimming out of the weir rooms, the arched stone dike (locals call them "stretched leg", with reference to the "leg" of the weir rooms) that links the weir rooms often has a half-curved spiral at its end: fish swimming along the puzzled dike are thus redirected back towards the weir rooms ( $\mathrm{Li}, 2014)$.

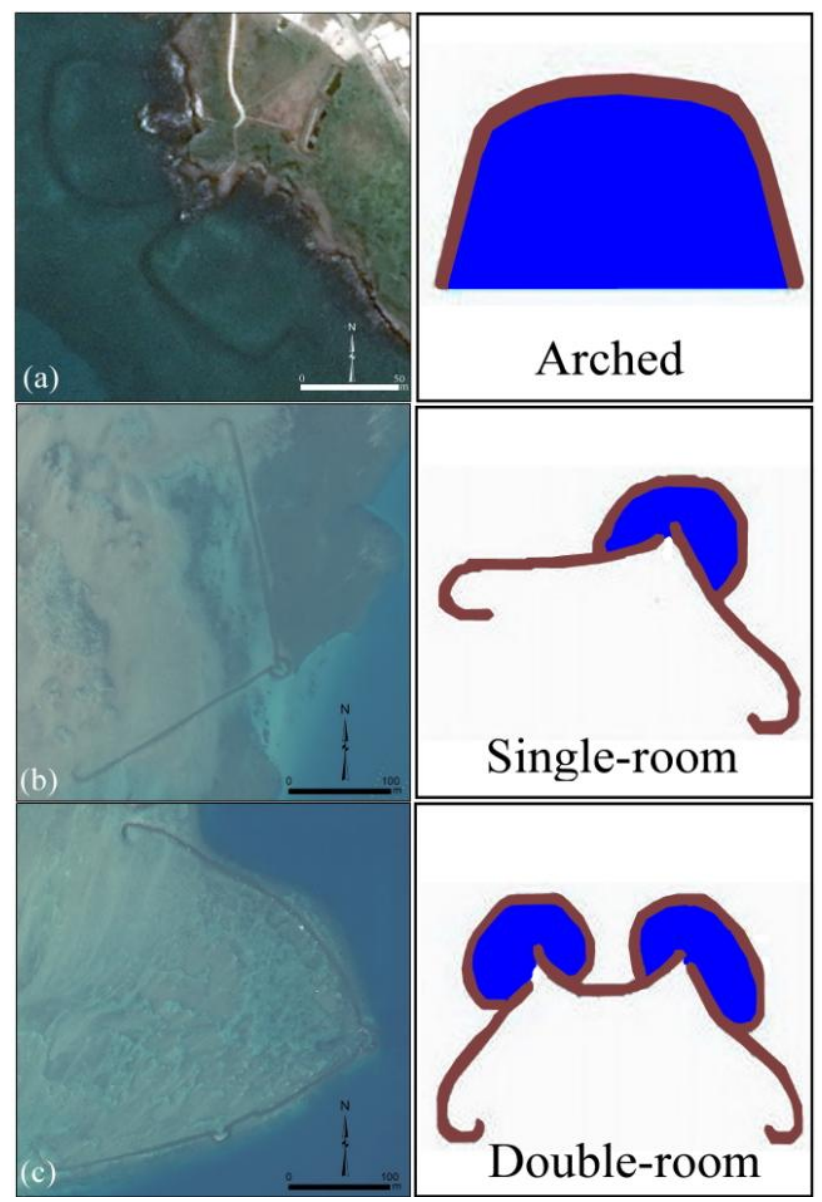

Figure 2. The left panel shows GoogleEarth images (@ GeoEye Image Copyright 2015, DigitalGlobe) of three typical STWs, and the right panel shows schematic views corresponding to the left images: a-Arched, b-Single-room, c-Double-room. 


\subsection{Distribution map and clusters}

The distribution map was made in ArcGIS10.1 by digitally drawing polyline features over STWs from GoogleEarth imagery, and polyline features were converted to points for the global mapping. Figure 3 shows a distribution map of the STWs.
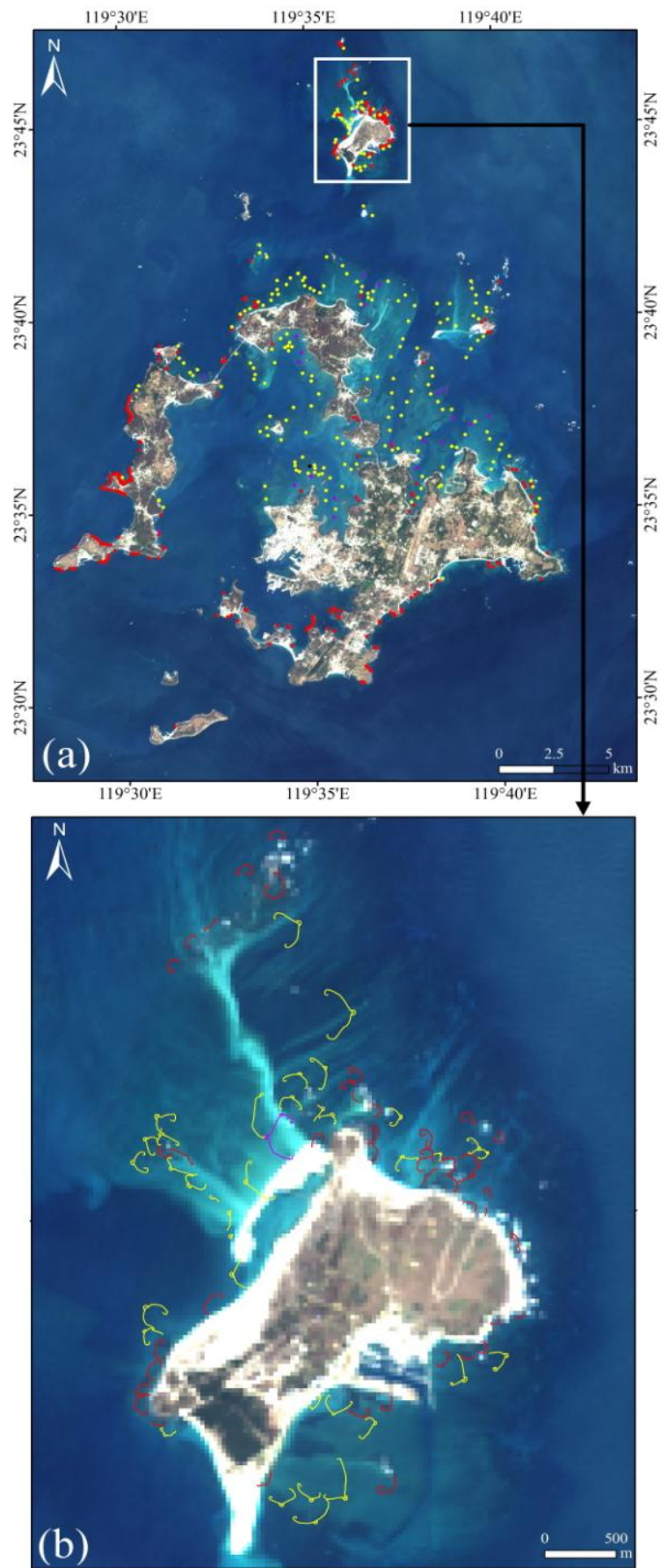

Figure 3. The distribution of STWs on Penghu and Chipei Island (the base map is the Landsat-8 OLI image which can be downloaded from http://www.usgs.gov), red, yellow and violet represent the arched, single-room and double room, respectively.

From spring to summer of 2015 our research team conducted investigations into Penghu's STWs based on different seasons and time periods of GoogleEarth historic images. Our results showed that, distributed amongst Penghu's coastline, there are 503 STWs.
The counts of Arched STWs, Single-room STWs, and Doubleroom STWs are 311, 254, and 27 in the reference catalog, respectively. In the Figure $3 \mathrm{a}$, the red points denote Arched STWs that our method has extracted and that are present in the reference catalog; there are 252 such STWs. Thus, we have extracted $81.0 \%$ of all STWs in the reference catalog. The yellow points denote Single-room STWs that our method has extracted and that are present in the reference catalog; there are 227 such STWs. Thus, we have extracted $89.4 \%$ of all STWs in the reference catalog. The violet points denote Double-room STWs that our method has extracted and that are present in the reference catalog; there are 24 such STWs. Thus, we have extracted $88.9 \%$ of all STWs in the reference catalog. The majority (59) of the missed STWs are Arched STWs, showing no sharp traces in the GoogleEarth imagery due to the seawater submersion, especially in the Xiyu Islands. An additional 27 Single-room and 3 Double-room STWs are mostly on the margin of the intertidal flats and are not extracted because they are not full outlines. Moreover, due to differing factors such as image quality (spatial resolution and acquisition date), STWs' shape and orientation, and tidal currents throughout the Penghu Islands. Table 1 summarizes a comparison between the reference catalog and the catalog produced by our method.

\begin{tabular}{|l|c|c|c|}
\hline Categories & \multicolumn{2}{|c|}{ Catalog } & Percent \\
\hline & reference (R) & GoogleEarth(G) & G/R*100\% \\
Arched & 311 & 252 & 80.0 \\
Single-room & 254 & 227 & 89.4 \\
Double-room & 27 & 24 & 88.9 \\
Total & 592 & 503 & 85.0 \\
\hline
\end{tabular}

Table 1. Comparison of STWs cataloged by GoogleEarth-based method with STWs in the reference catalog.

\subsection{Lengths of STWs}

By using the geometry calculator in ArcGIS10.1, the length of the STWs in Penghu Islands range from $10 \mathrm{~m}$ to hundreds of meters, some great STWs even was surpassing $500 \mathrm{~m}$ or even $1,000 \mathrm{~m}$. Table 2 shows length segmented counts for different STWs. Penghu Islands has 117 great STWs, including 103 STWs with length range from $500 \mathrm{~m}$ to $999 \mathrm{~m}$ and 14 STWs with length more than $1,000 \mathrm{~m}$. In this study, the lengths of the largest Arched STWs, Single-room STWs, and Double-room STWs are $487 \mathrm{~m}, 1,259 \mathrm{~m}$, and 1,204 m respectively.

\begin{tabular}{|l|c|c|c|c|c|}
\hline Categories & \multicolumn{5}{|c|}{ Counts } \\
\hline Length/m & $0-99$ & $100-299$ & $300-499$ & $500-999$ & $1,000-$ \\
Arched & 53 & 183 & 16 & 0 & 0 \\
Single-room & 4 & 45 & 79 & 89 & 10 \\
Double-room & 0 & 2 & 4 & 14 & 4 \\
Total & 57 & 230 & 99 & 103 & 14 \\
\hline
\end{tabular}

Table 2. Length segmented counts of GoogleEarth-based extracted STWs in Penghu Islands.

\subsection{Functions and culture}

Compared with other methods of fish catching, such as netting and poisoning, the use of STWs has an incomparably positive advantage to the preservation of marine ecology and environment. STWs follow the rhythm of the tides to catching fish; unlike the use of nets, there is no problem of overfishing. The stone materials used to build STWs are entirely natural, without concrete or other man-made substances and chemicals that can contribute to coastal and maritime pollution. Moreover, 
the inside of these weirs is actually a vigorous breeding pool for various marine life forms, including young fish, crabs, and shrimp (Li, 2014). Furthermore, STWs on the inter-tidal flats can resist wind and waves, and even typhoons. Finally, the construction of STWs not only contributes to the preservation of marine life, but acts as a wave breaker, helping to stem land erosion ( $\mathrm{Li}, 2014)$.

Constructing a STW would have often required the labor of an entire clan, community, or whole village; and the subsequent management and operation of a weir would be a vital matter for the entire community. Also, the amount of fish caught depended on the weir's position, technology, scale, and even supernatural powers. Thus, the fishing, maintenance, distribution, inheritance, pledge mechanisms and even rituals of the community revolved entirely around the STWs, which took on religious connotations, with villagers worshiping the weir and praying to the specific gods and the religious faith that grew out of it ( $\mathrm{Li}, 2014)$. The STWs held life together for fishing societies, becoming an important feature of island culture in Penghu archipelago.

\section{CONCLUSIONS}

Stone tidal weir, an oldest fishing trap on our planetary, was once widely distributed in coastal areas with remarkable tidal action and coral reef areas with developed lagoons all over the world. It is normally a dustpan-shaped or necklace-shaped made of stones or coral lime stones. And its construction has a close relationship to tidal change. The fish swim or are driven into the weir during high tide but cannot find their way out during low tide. Different architectural styles of stone weirs were attributed to different cultures, who shared a mixed piscatorial and agricultural economic base and a fish centred world-view.

This large-scale archaeological investigation of the STWs illustrates the Google Earth, as sources of freely-available, high resolution imagery, is a powerful tool for cultural heritages and geoarchaeological research. This article evaluates the utility of Google Earth images to identify linear archaeological traces of STWs and integrates them in a GIS to produce a distribution map of STWs in Penghu archipelago. Not less than 503 STWs, $85.0 \%$ of all STWs in the reference catalog, were extracted based on our proposed method which is more time-saving and efficient.

Due to the ever-increasing population pressure, the demand for fish catches increases by the day in modern times. The STWs cannot meet the demand lead to this traditional method of waiting idly for fishes to come now seems outdated; this, combined with technological innovation, skilled labor, and innovative equipment has meant the abandonment of STWs ( $\mathrm{Li}$, 2014). Yet, as a precious cultural heritage and a symbol of fishing civilizations, these mysterious necklaces scattered on the intertidal flats will forever deserve our admiration, preservation, and treasuring.

\section{ACKNOWLEDGEMENTS}

Many thanks are given to the GoogleEarth for providing the very high resolution imagery that were used in this paper.

\section{REFERENCES}

Alexakis, D., Sarris, A., Astaras, T., Albanakis, K., 2011. Integrated GIS, remote sensing and geomorphologic approaches for the reconstruction of the landscape habitation of Thessaly during the Neolithic period. J. Archaeol. Sci., 38(1), pp. 89-100.

Espa, G., Benedetti, R., De Meo, A., Ricci, U., Espa, C., 2006. GIS based models and estimation method for the probability of archaeological site location. J. Cult. Herit., 7(3), pp. 147-155.

Evans, D., Pottier, C., Fletcher, R., Hensley, S., Tapley, I., Milne, A., Barbetti, M., 2007. A comprehensive archaeological map of the world's largest preindustrial settlement complex at Angkor, Cambodia. Proc. Natl. Acad. Sci. U. S. A., 104, pp. 14277-14282.

Hong, G., 1999. The Stone Tidal Weirs in Penghu Islands. Penghu Hsien Cultural Centre, Penghu, pp. 48-218 (in Chinese).

Kempe, S., Al-Malabeh, A., 2013. Desert kites in Jordan and Saudi Arabia: Structure, statistics and function, a Google Earth study. Quat. Int., 297, pp. 126-146.

Li, M., 2009. The Origin and Distribution of Fish Weir Culture, An Exploration with an International Perspective. Cultural Affairs Bureau, Penghu County, Penghu, pp. 52-64 (in Chinese).

Li, M., 2014, Stone Weirs of Penghu Islands. China Scenic, 5, pp. 109-118.

Li, M., Zhan, Y., 2006, Digital Archives Program of Penghu Stone Tidal Weirs. Coral Stone, 44, pp. 2-19 (in Chinese).

Luo, L., Wang, X., Liu, C., Guo, H., Du, X., 2014, Integrated RS, GIS and GPS approaches to archaeological prospecting in the Hexi Corridor, NW China: A case study of the royal road to ancient Dunhuang. J. Archaeol. Sci., 50, pp. 178-190.

Sadr, K., Rodier, X., 2012. Google Earth, GIS and stone-walled structures in southern Gauteng, South Africa. J. Archaeol. Sci., 39(4), pp. 1034-1042. 